\title{
TOWARD INTEGRATED MULTI-SCALE SIMULATIONS OF FLOW AND SEDIMENT TRANSPORT IN RIVERS
}

\author{
Shoji FUKUOKA ${ }^{1}$ and Tatsuhiko UCHIDA ${ }^{2}$ \\ 1 Fellow of JSCE, PhD., Dr. of Eng., Professor, Research and Development Initiative, Chuo University \\ (1-13-27 Kasuga, Bunkyo-ku, Tokyo 112-8551, Japan) \\ 2 Member of JSCE, Dr. of Eng., Associate Professor, Research and Development Initiative, Chuo University (ditto)
}

\begin{abstract}
An integrated multi-scale simulation method of flow and sediment transport in rivers is required for river channel designs and maintenance managements. This paper highlights the need of a new depth integrated model without the assumption of the shallow water flow for integrated simulations of $2 \mathrm{D}$ and 3D phenomena in rivers, indicating the applicable ranges and limitations of previous depth integrated models. The general Bottom Velocity Computation method without the shallow water assumption is proposed and its applicability for flows with $3 \mathrm{D}$ structures and bed variations is discussed in this paper. The unified calculation method for integrated multi-scale simulations is presented to elucidate flow and sediment transport in laboratory channel and rivers during flood. Finally, we discuss issues to be solved and the future works toward integrated multi-scale simulations of flow and sediment transport in rivers.
\end{abstract}

Key Words: integrated multi-scale simulation, depth integrated model, shallow water assumption, flood observation, phenomena scale in river

\section{INTRODUCTION}

Flows and sediment transport are controlled by two kinds of boundaries confining the river width in the lateral direction and the water depth in the vertical directions. River engineers and researchers have applied one-dimensional analysis method for the objective scale which is much larger than the river width, two-dimensional analysis method for the problem of the phenomena scales between the river width and the water depth, and three-dimensional analysis method for the target phenomena much smaller than the water depth ${ }^{1), 2)}$. Fig. 1 shows various phenomena scales of flow and sediment transport in rivers and their calculation methods. Because many important phenomena related to the river management and design are affected by the large scale phenomena compared with the water depth, many calculation methods based on 2D models have been developed for practical problems in river ${ }^{1-5)}$. On the other hand, smaller scale phenomena than the water depth, 3D flow structures, can affect larger scale phenomena through bed variations and flow resistances. Although simulations of complex turbulence flows and local scouring have been made possible by recent advanced 3D turbulence models 1),2),

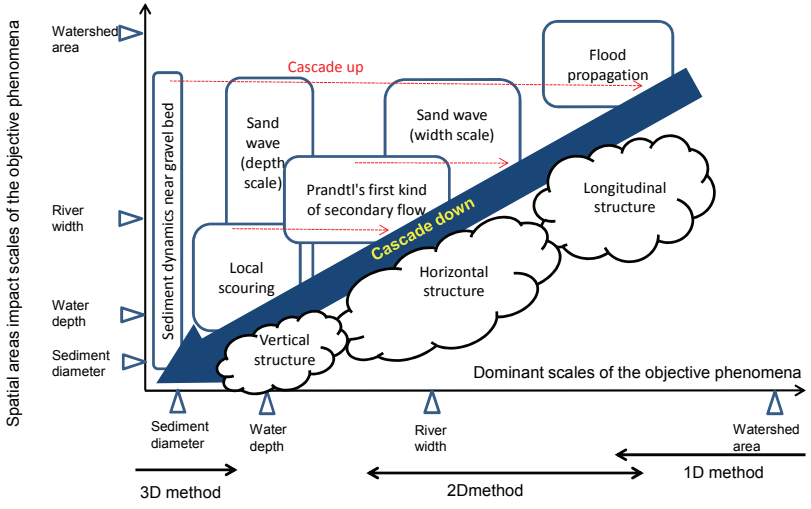

Fig. 1 Scales and calculation methods of various phenomena in rivers.

applications of the 3D model are still limited to small scale phenomena such as flows and bed variations in experimental channels, because of large computational time, many memories, and the huge computational task.

For an integrated multi-scale simulation method of flow and sediment transport in rivers, a new calculation method is necessary to complement the $2 \mathrm{D}$ and $3 \mathrm{D}$ models. This paper indicates the applicable ranges and limitations of previous models, and then highlights the need of a new depth integrated 


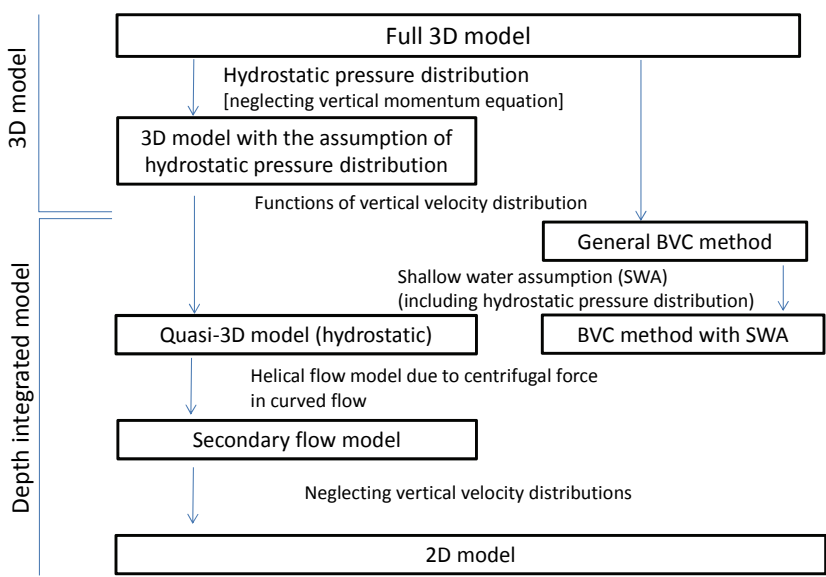

Fig. 2 Various types of depth integrated model including bottom velocity computation method.

model without the assumption of the shallow water flow. The present integrated simulation method is based on the general Bottom Velocity Computation method without the shallow water assumption. We apply and verify the method to flows and bed variations in laboratory channels, which were difficult phenomena to be calculated by previous depth integrated models. This paper also presents a calculation method combined with observation data to elucidate flow and sediment transport in rivers during a flood. Finally, we discuss issues and future works.

\section{VARIOUS DEPTH INTEGRATED MODELS AND THEIR APPLICABILITY RANGE}

An advanced depth integrated model, an improved 2D model, is one of the effective methods to compute large-scale horizontal flow phenomena with considering 3D flow effects. Many improved depth integrated models have been developed especially for a helical flow in a curved or meandering channel, as shown in Fig. 2. Those include fully developed secondary flow model ${ }^{6,7)}$ without considering effects of secondary flow on vertical distribution of stream-wise velocity, and refined secondary flow models with non-equilibrium secondary flow $^{8,9)}$ and mean flow redistribution effects ${ }^{10,11)}$. A quasi-3D model is a more versatile method to evaluate vertical velocity distributions by depth integrated equations. Equations for vertical velocity distributions for quasi-3D models have been derived from the 3D momentum equation by using a weighted residual method ${ }^{12-17)}$. Many quasi-3D models employ the assumption of the hydrostatic pressure distribution ${ }^{12-15)}$, because the non-hydrostatic quasi-3D model based on the weighted residual method ${ }^{10,17)}$ is difficult to be solved.

To dissolve the limitations of the previous depth integrated models, we have developed a new quasi-3D method, the
Bottom Velocity Computation (BVC) method. As shown in Fig.2, bottom velocity computation methods are distinguished by whether the shallow water flow is assumed ${ }^{18)}$ or not ${ }^{19 \nmid-21)}$.

The important equation of the BVC method is the bottom velocity equation (1), which is derived by depth-integrating horizontal vorticity.

$$
\delta u_{i}=u_{s i}-u_{b i}=\varepsilon_{i j 3} \Omega_{j} h+\frac{\partial W h}{\partial x_{i}}-w_{s} \frac{\partial z_{s}}{\partial x_{i}}+w_{b} \frac{\partial z_{b}}{\partial x_{i}}
$$

Where, $u_{b i}$ : bottom velocity, $u_{s i}$ : water surface velocity, $\Omega_{j}$ : depth averaged (DA) horizontal vorticity, $h$ : water depth, $W$ : DA vertical velocity, $z_{s}$ : water level, $z_{b}$ : bed level, $w_{s}, w_{b}$ : vertical velocity on water surface and bottom. The bottom shear stress acting on the bed $\tau_{b i}$ is evaluated with equivalent roughness $k_{s}$, assuming the equilibrium velocity condition in the bottom layer $\delta z_{b}^{21)}$.

$$
\tau_{b i}=\left(c_{b}\right)^{2} u_{i} u_{b}, \frac{1}{c_{b}}=A r+\frac{1}{\kappa} \ln \left(\frac{\delta z_{b}+a k_{s}}{k_{s}}\right)
$$

Where, $h / \delta z_{b}=e^{3}-1, a=1$. On the other hand, the bottom pressure intensity is given by Eq.(3), depth-integrating the vertical momentum equation.

$$
\frac{d p_{b}}{\rho}=\frac{\partial h W}{\partial t}+\frac{\partial h W U_{j}}{\partial x_{j}}+\tau_{b j} \frac{\partial z_{b}}{\partial x_{j}}-\frac{\partial h \tau_{z j}}{\partial x_{j}}
$$

Where, $d p$ : pressure deviation from hydrostatic pressure distribution $\left(p=\rho g\left(z_{s}-z\right)+d p\right), d p_{b}$ : $d p$ on bottom, $\tau_{b j}$ : bed shear stress, $\tau_{i j}$ : shear stress tensor due to molecular and turbulence motions and vertical velocity distribution. To calculate Eqs. (1) and (3), the governing equations for the following unknown quantities are solved: water depth $h$ (Eq.(4)), DA horizontal velocity $U_{i}$ (Eq.(5)), DA turbulence energy $k$ (Eq.(6)), DA horizontal vorticity $\Omega_{i}$, (Eq.(7)) horizontal velocity on water surface $u_{s i}$ (Eq.(8)), DA vertical velocity $W$ (Eq.(9)).

$$
\begin{aligned}
& \frac{\partial h}{\partial t}+\frac{\partial U_{j} h}{\partial x_{j}}=0 \\
& \frac{\partial U_{i} h}{\partial t}+\frac{\partial U_{i} U_{j} h}{\partial x_{j}}=-g h \frac{\partial z_{s}}{\partial x_{i}} \\
& -\frac{\partial h d p_{0}}{\rho \partial x_{i}}-\frac{d p_{b}}{\rho} \frac{\partial z_{b}}{\partial x_{i}}-\frac{\tau_{b i}}{\rho}+\frac{\partial h \tau_{i j}}{\rho \partial x_{j}} \\
& \frac{\partial k}{\partial t}+U_{j} \frac{\partial k}{\partial x_{j}}=\frac{1}{h} \frac{\partial}{\partial x_{i}}\left(\frac{v h}{\sigma_{k}} \frac{\partial k}{\partial x_{i}}\right)+P_{k}-\varepsilon \\
& \frac{\partial \Omega_{i} h}{\partial t}=R_{\sigma i}+P_{\omega i}+\frac{\partial h D_{\omega i j}}{\partial x_{j}} \\
& \frac{\partial u_{s i}}{\partial t}+u_{s j} \frac{\partial u_{s i}}{\partial x_{j}}=-\left\{g+\left(\frac{\partial d p}{\partial z}\right)_{z=z_{s}}\right\} \frac{\partial z_{s}}{\partial x_{i}}+P_{s i}
\end{aligned}
$$



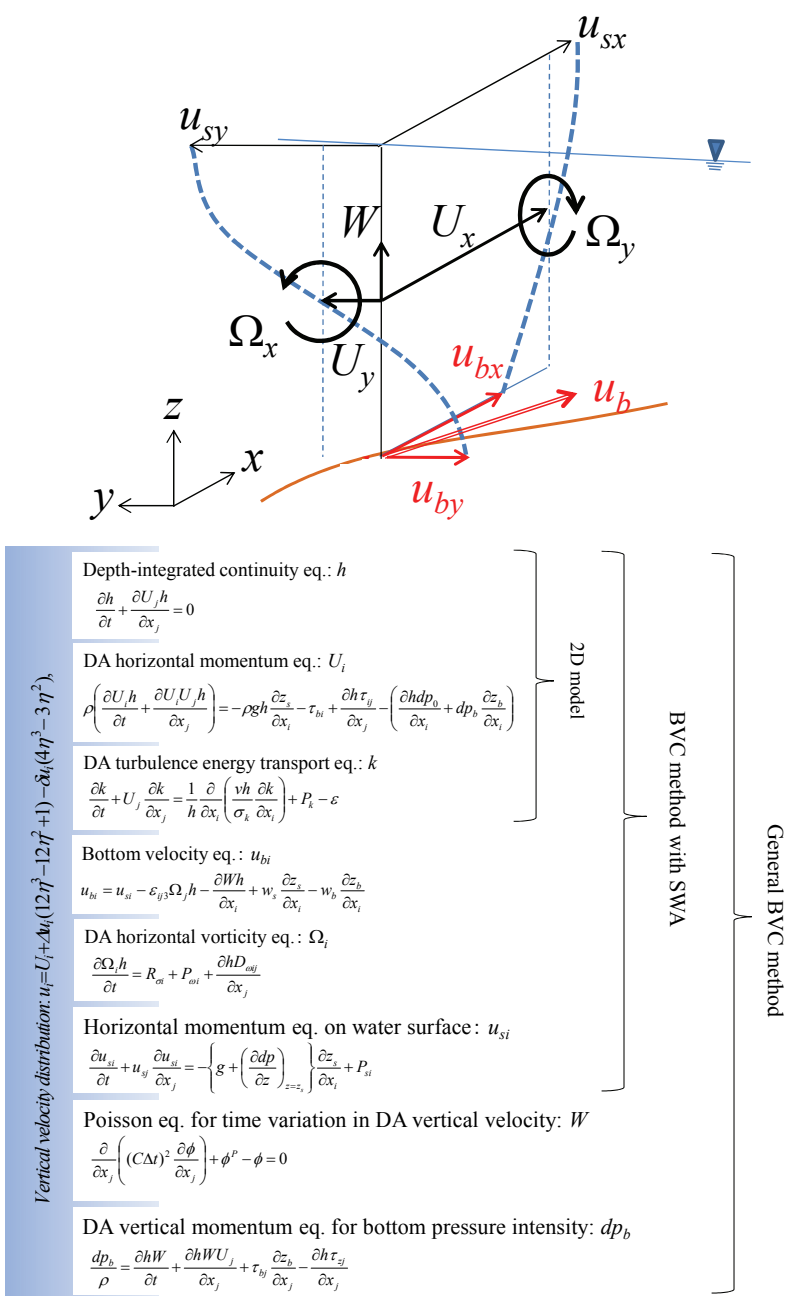

DA: Depth Averaged, BVC: Bottom Velocity Computation, SWA: Shallow Water Assumption $\Delta u_{i}=u_{\mathrm{si}}-U_{i}, \boldsymbol{\alpha}_{i}=u_{i j}-u_{b_{i j}}$

Fig.3 Governing equations and unknown quantities of BVC method

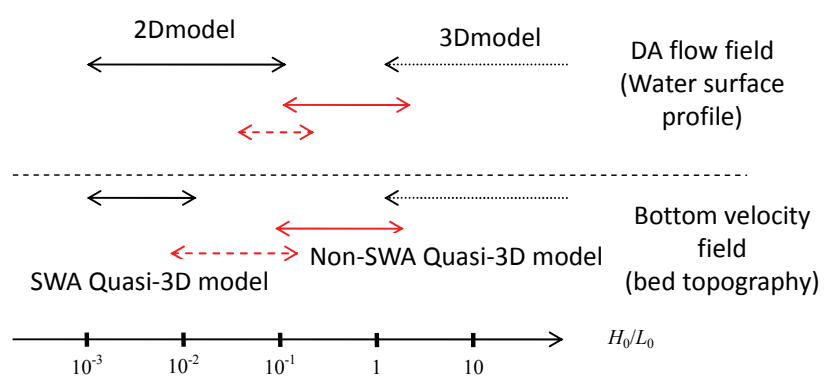

Fig. 4 Limitations of various models for shallowness parameter of objective phenomena

$$
\frac{\partial}{\partial x_{j}}\left((C \Delta t)^{2} \frac{\partial \phi}{\partial x_{j}}\right)+\phi^{P}-\phi=0
$$

Where, $d p_{0}$ : depth-averaged $d p, \sigma_{k}=1, v=v_{m}+v_{t}, v_{m}$ : kinematic viscosity coefficient, $v_{t}$ : kinematic eddy viscosity coefficient, $P_{k}$ : the production term of turbulence energy, $\varepsilon$ : the dissipation term of turbulence energy, $R_{\sigma i}$ : the rotation term of vertical vorticity $\left(R_{\sigma i}=u_{s i} \omega_{\sigma s}-u_{b i} \omega_{\sigma b}\right), \omega_{\sigma s,} \omega_{\sigma b}$ : rotation of $u_{s i}, u_{b i}, P_{\omega i}$ : the production term of horizontal vorticity, $D_{\omega i j}$ : horizontal vorticity flux due to convection, rotation, dispersion and

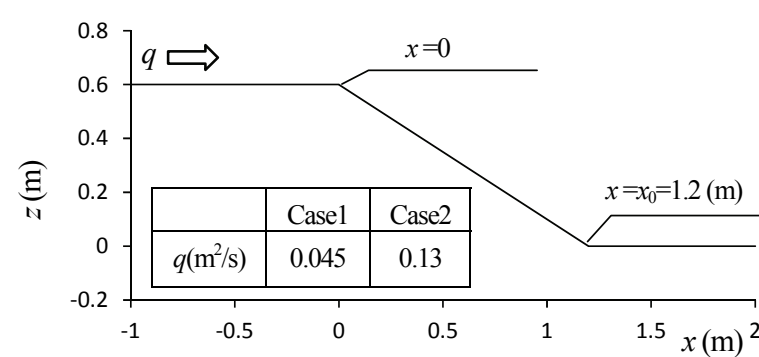

Fig.5 The experimental conditions on rapidly varied flow over a structure $^{22)}$

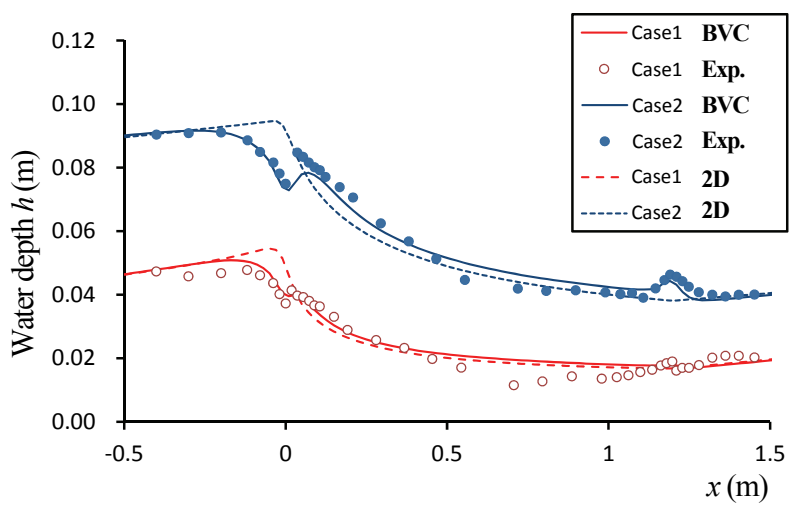

Fig.6 Longitudinal changes in water depth for rapidly varied flow over a structure $^{20)}$

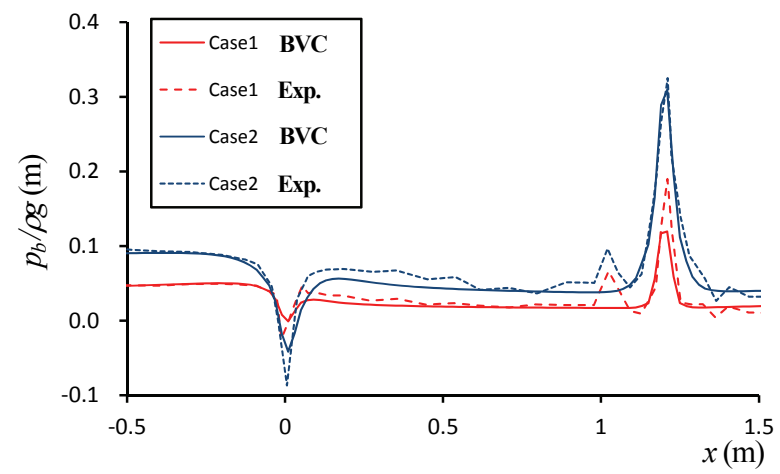

Fig.7 Longitudinal changes in bottom pressure intensity for rapidly varied flow over a strucutre ${ }^{20)}$

turbulence diffusion, $P_{s i}$ : the production term of surface velocity (shear stress under the water surface layer), $C=k_{1} h / \Delta t, \quad k_{1}=1 / 20$, $\phi=(W h)^{n+1}-(W h)^{n}, \phi^{P}=(W h)^{P}-(W h)^{n},(W h)^{P}$ : the predicted $W h$ calculated by the continuity equation using $\left(\delta u_{i}\right)^{P},\left(\delta u_{i}\right)^{P}$ : predicted velocity difference calculated by Eq (1) with $(W h)^{n}$. These equations are obtained by assuming vertical velocity distributions. The BVC method is summarized in Fig.3. Refer to the literatures ${ }^{18)-21)}$ for details of the equations and the numerical computation methods for the BVC method.

To clarify the applicability ranges and limitations of various numerical methods in Fig.2, we have investigated magnitude of each term in depth averaged momentum equation (5) for depth averaged flow analysis and bottom velocity equation (1) for bed variation analysis ${ }^{19)}$. Fig.4 shows summary of the results ${ }^{19)}$ and limitations of various models for the shallowness parameter $\varepsilon_{S}$ ( $\varepsilon_{s}=h_{0} / L_{0}<<1, h_{0}$ : the representative vertical scale (water depth), $L_{0}$ : the representative horizontal scale of objective phenomena). 
Table 1 Experimental hydraulic conditions and the various bed forms ${ }^{26)}$.

\begin{tabular}{|c|c|c|c|c|c|c|}
\hline & & & $d$ & & Bedfo & type \\
\hline & 1 & $\left(\mathrm{~m}^{2} / \mathrm{s}\right)$ & (m) & $T r$ & Exp. & Cal. \\
\hline 1 & $1 / 178$ & $41.8 * 10^{-3}$ & $0.76^{*} 10^{-3}$ & 0.66 & Dune & Dune \\
\hline 2 & $1 / 147$ & $43.0^{*} 10^{-3}$ & $0.76^{*} 10^{-3}$ & 0.65 & Duen & Dune \\
\hline 3 & $1 / 73.0$ & $44.3 * 10^{-3}$ & $0.76^{*} 10^{-3}$ & 0.88 & $\begin{array}{c}\text { Anti-dune } \\
\text { moving } \\
\text { downstream }\end{array}$ & $\begin{array}{c}\text { Dune with } \\
\text { long wave } \\
\text { length }\end{array}$ \\
\hline 4 & $1 / 31.0$ & $39.5 * 10^{-3}$ & $0.76^{*} 10^{-3}$ & 1.74 & Plane bed & Plane bed \\
\hline 5 & $1 / 31.0$ & $10.8 * 10^{-3}$ & $0.19^{*} 10^{-3}$ & 1.11 & Anti-dune & Anti-dune \\
\hline 6 & $1 / 31.0$ & $26.8 * 10^{-3}$ & $0.19 * 10^{-3}$ & 1.24 & Anti-dune & Anti-dune \\
\hline 7 & $1 / 20.0$ & $13.3 * 10^{-3}$ & $0.19^{*} 10^{-3}$ & 0.65 & Anti-dune & Anti-dune \\
\hline
\end{tabular}

$I$ : channel slope, $q$ :discharge per unit width, $d$ : sediment particle diameter, $F r$ : averaged Froude number (Local Fr varies longitudinally especially for case $5 \sim 7$.)

For depth averaged (DA) flow fields, the applicability range of the $2 \mathrm{D}$ model covers wide ranges of the shallowness parameter. On the other hand, for bottom velocity fields, the applicability of the $2 \mathrm{D}$ model is limited to the narrow range of the shallowness parameter due to variations in vertical velocity distributions. This means that a quasi-3D model plays a more significant role in bed variation analysis. However, the shallow water assumption is unsuitable for $0.1<\varepsilon_{s}$, because the magnitude of spatial difference terms in vertical velocity of Eq.(1) and non-hydrostatic pressure terms of the horizontal momentum equation (5) and the water surface momentum equation (8) becomes larger with increasing $\varepsilon_{s}$. For above results, a quasi-3D model without the shallow water assumption is required for multi-scale simulations of flow and sediment transport in rivers.

\section{APPLICATIONS OF THE GENERAL BVC METHOD TO FLOW AND SEDIMENT TRANSPORT IN A NARROW CHANNEL}

\section{(1) Rapidly varied flow over a structure}

A calculation method for flow over a structure is required for many practical problems with respect to flood and inundation flows. However, it is difficult to evaluate rapidly varied flow by many depth integrated model because of the vertical acceleration with the non-hydrostatic pressure distribution. This section discusses the ability of the general BVC method for calculating rapidly varied flows over a structure. Fig.5 shows hydraulic conditions of the experiments ${ }^{22)}$. Boundary conditions of upstream end for calculations are experimental discharge and water depth, for the state of flow over the structure was a super critical flow.

Fig.6 and Fig.7 show comparisons of longitudinal distributions of water depth and bottom pressure intensity between the experiment ${ }^{22)}$ and calculation ${ }^{20)}$ by the general BVC method, respectively. Fig.6 also shows results by the 2D

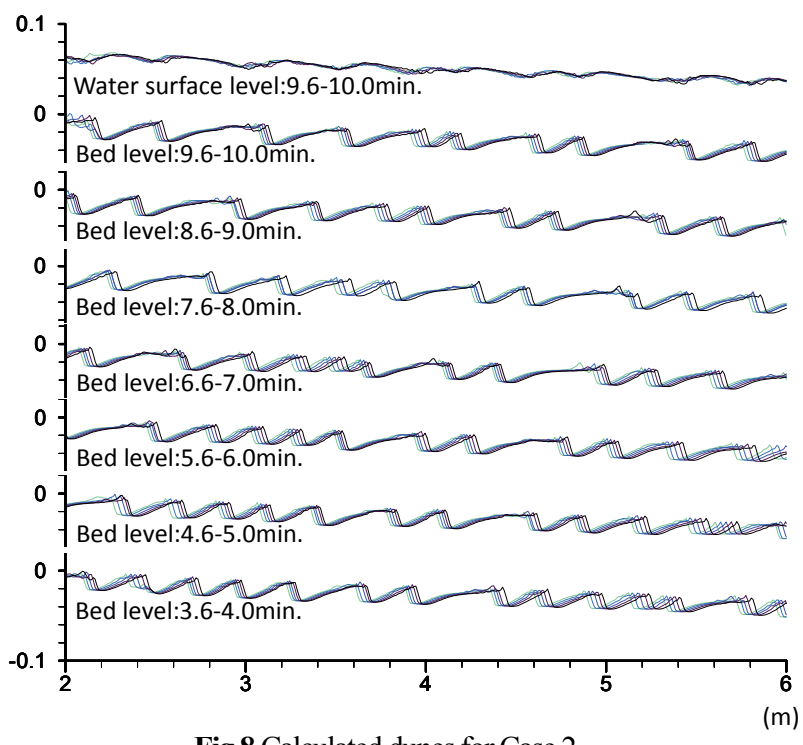

Fig.8 Calculated dunes for Case 2

method. We can see the abrupt drop in the bottom pressure intensity at $x=0$ in Fig.7. For Case2, the negative pressure intensity was measured there. Although the flow is supercritical, the water depth for $x<0$ decreases due to the change in slope at $x=0$. On the other hand, water depth increases due to pressure rising produced by the stream curvature at $x=1.2$. These phenomena caused by non-hydrostatic pressure cannot be calculated by the 2D method, as shown in Fig.6. It is demonstrated that the general BVC method can calculate longitudinal profiles of water depth and pressure intensity acting on the bed for rapidly varied flows over a structure, as shown in Fig.6 and Fig.7.

(2) Flow resistance and sediment transport with sand waves

The generation of sand waves complicates behaviors of flood flow, rate of sediment transport and structure of bed topography in rivers. Recently, some researchers have explained the patterns of dunes in a narrow experimental channel by using a vertical 2D turbulence model with a sediment transport model $^{23), 24)}$. However, it would be unrealistic to apply these models to $3 \mathrm{D}$ flows and bed morphology in rivers due to extremely high computational cost. A few researchers have tried to calculate these phenomena by a depth integrated model $^{25)}$. In this section, the applicability of the general BVC method for various sand wave calculations is discussed. Table 1 shows experimental conditions with a variety of channel slopes, discharges and particle size diameters. Those are a part of experimental conditions on various bed forms conducted by Fukuoka et $a l^{26}{ }^{26}$. Boundary conditions of the upstream end for calculations are given by experimental discharge. The shear stresses acting on the bed and side wall are evaluated with equivalent roughness $k_{s}=d$ ( $d$ : sediment particle diameter) and Manning's roughness coefficient $n=0.008$, respectively. The time variation in the bed level is calculated by the $2 \mathrm{D}$ continuity 


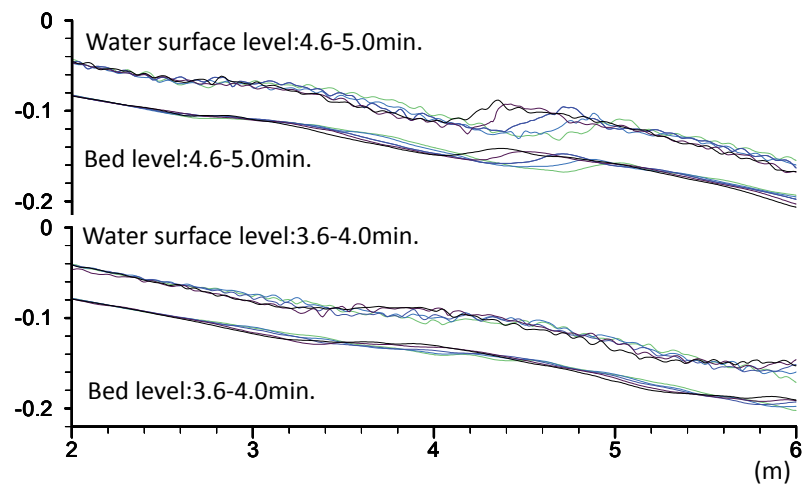

Fig.9 Calculated plane beds for Case 4

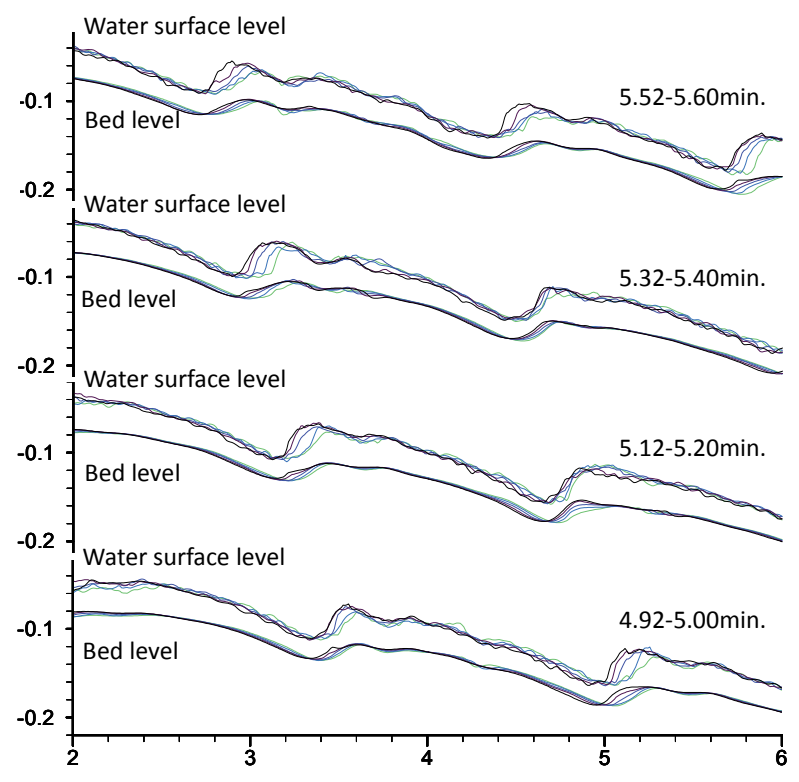

Fig.10 Calculated anti-dunes for Case 6

equation for sediment transport of the Exner's form. The momentum equation for sediment motion ${ }^{18)}$ is used to evaluate non-equilibrium sediment transport rate. The details of the calculation method are discussed in the literature ${ }^{21)}$.

Table 1 describes types of calculated bed forms for different hydraulic conditions. Fig.8-10 show time variations in water surface and bed profiles. Each figure shows 5 snapshots at the constant time interval for the duration indicated in the figure. Although the regime of the anti-dune moving downstream, which was seen in the experimental channel, could not be reproduced, the three different types of bed forms, dune (Fig.8), plane bed (Fig.9) and anti-dune (Fig.10) were reproduced by the general BVC method. We can see that development of dunes moving downstream with coalescence (Fig.8) and antidunes with intermittent hydraulic jumps (Fig.10). Fig.11 shows comparisons of measured and calculated water depth and sediment discharge for Case 1 7. The water depths and sediment discharge rates in the experimental channel with different bedform types are reproduced by the general BVC method.

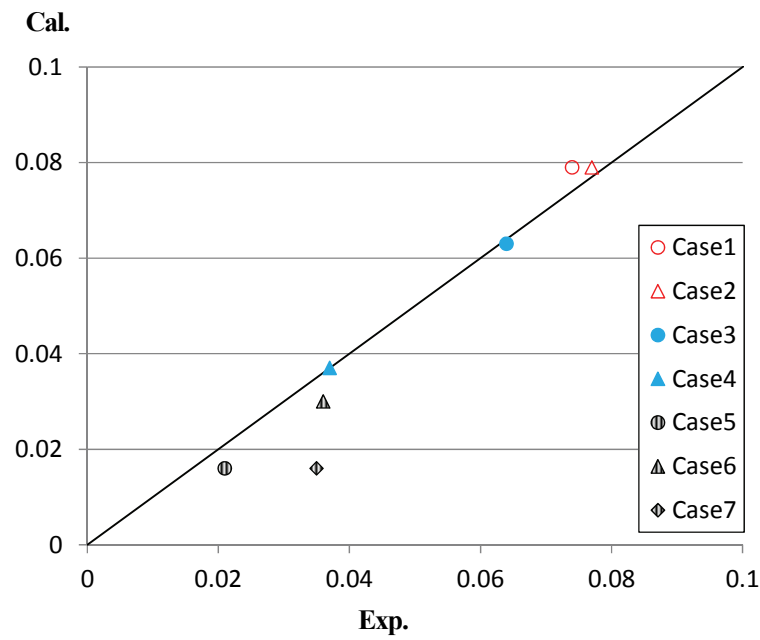

(1) Water depth (m)

Cal.

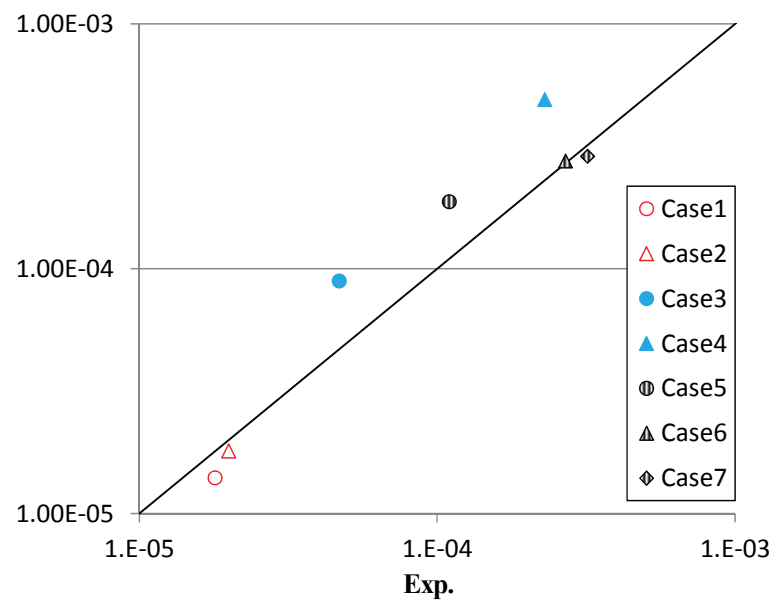

(2) Sediment discharge rate per unit width $\left(\mathrm{m}^{2} / \mathrm{s}\right)$

Fig.11 Comparisons of water depth and sediment discharge for various hydraulic conditions with sand waves

\section{APPLICATIONS OF THE GENERAL BVC METHOD TO 3D FLOW AND BED VARIATIONS}

(1) 3D flow structures around a cylinder

Many studies have been conducted to clarify the pier scour mechanisms. However, few depth integrated models have been proposed for complex 3D flows with the horseshoe vortex in front of the structure. We investigate the applicability of the general BVC method for the local flow around the cylinder. The method is applied to the experimental results ${ }^{27)}$ for the flow around a cylinder on the fixed rough bed. Table 2 shows the experimental conditions. The size of computational gird around the cylinder was $1 / 40$ of the diameter. Experimental discharge is given at the upstream end and water depth is given at downstream end. The present computational method improves our previous computation ${ }^{19)}$ in evaluating bottom vorticity.

Fig. 12 shows instantaneous computed results of (a) depth- 
Table 2 Experimental conditions by Roulund et al. ${ }^{27)}$

\begin{tabular}{|c|c|}
\hline Cylinder diameter $(\mathrm{m})$ & 0.536 \\
\hline Depth $(\mathrm{m})$ & 0.54 \\
\hline Channel width $(\mathrm{m})$ & 4.0 \\
\hline Fr number & 0.14 \\
\hline
\end{tabular}

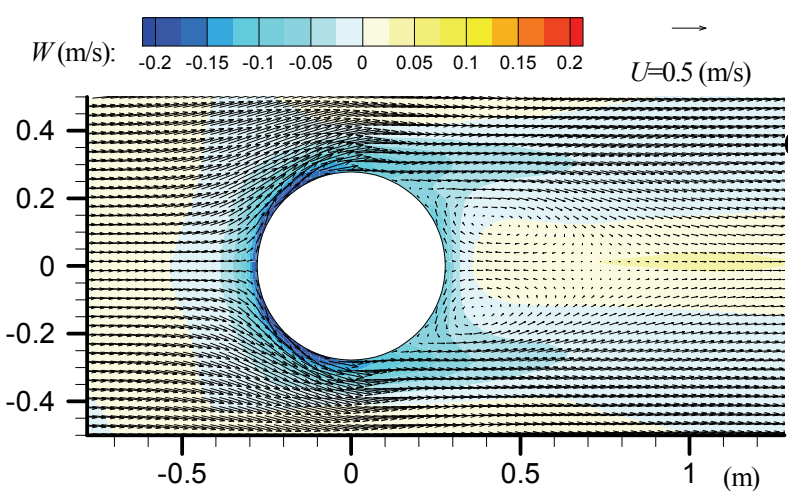

(a) Depth averaged velocity

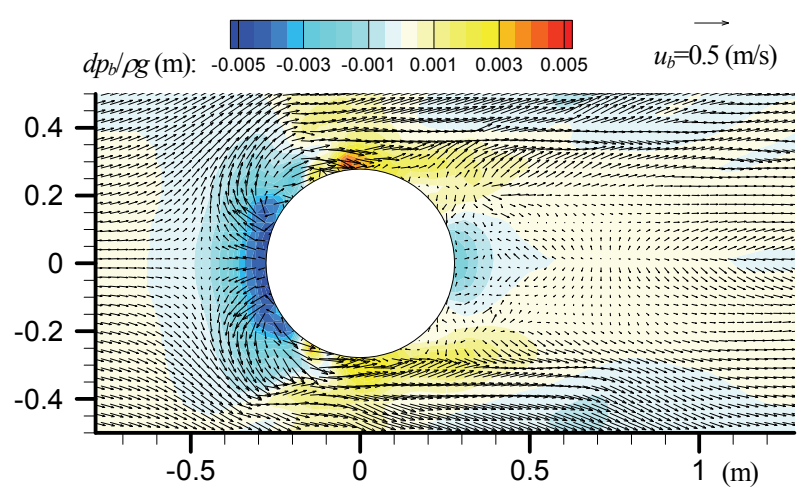

(b) Bottom velocity and non-hydrostatic bottom pressure

Fig.12 Calculation results by the general BVC method for flow around a cylinder in open channel flow

averaged velocity, (b) bottom velocity and non-hydrostatic pressure distributions. We can see downward and reverse bottom flows induced with low pressure in front of the cylinder and high pressure in both sides of the cylinder. Those are typical characteristics of the horse shoe vortex.

Fig. 13 compares calculation results with the experimental results for velocity distributions on the longitudinal crosssection along the center line of the cylinder. The opposite rotation vortex was calculated behind the cylinder compared to measured result. This seems to be caused by the over production of turbulent energy in the separation zone by the conventional turbulent model: unsteady turbulent flows with the Karman Vortex behind the cylinder were not developed in this calculation as shown in Fig.12. However, the calculation result in front of the cylinder provided a good agreement with the measurement result, which is important to calculate local scouring at the bed. Although the calculation method in the separation zone is still remained as issue, it assures to simulate
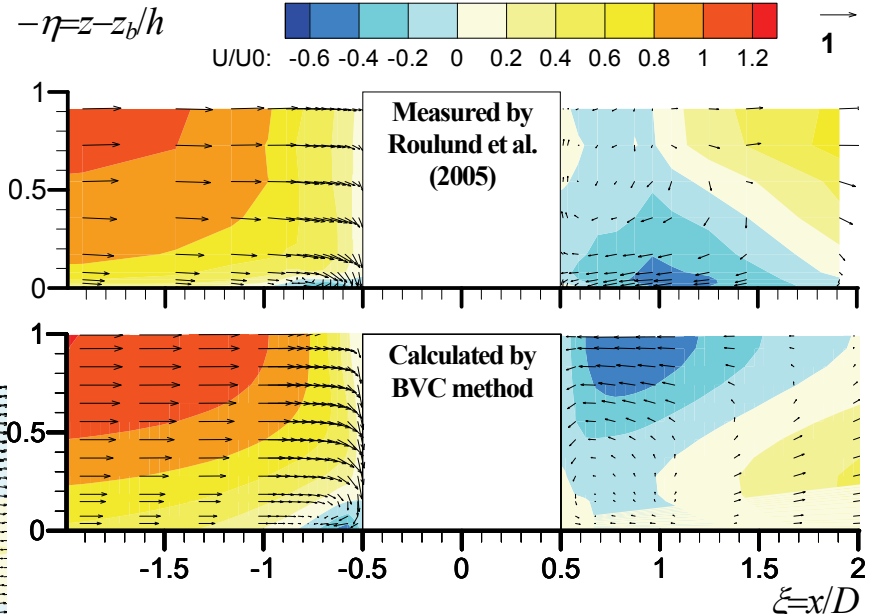

Fig.13 Non dimensional vertical velocity distributions along the center line of a cylinder.

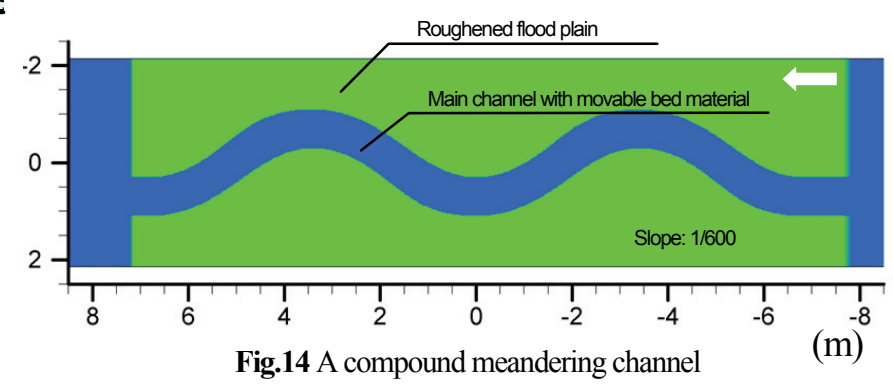

3D flow fields in front of a structure.

(2) Bed variation in a compound-meandering channel

In a compound-meandering channel, flow patterns change with increasing relative depth $D r=h_{p} / h_{m}$ ( $h_{p}$ : water depth on flood plains, $h_{m}$ : water depth on a main channel) due to the momentum exchange with vortex motions formed between high velocity flow in a main channel and low velocity flow in flood plains ${ }^{1) 28)}$. For this complex hydrodynamic phenomena, bed variation in a compound-meandering channel could not been explained by the depth integrated models because of their assumption of the shallow water flow. In this section, we apply the general BVC method coupled with a non-equilibrium sediment transport model $^{18)}$ to bed-variation analysis in a compound meandering channel. Fig.14 shows the experimental channel, which has a meandering main channel with movable sand bed and roughened flood plains. The bed variations in the main channel for various relative depths were investigated in the experiment ${ }^{28)}$. Bed variations in the flows of $\mathrm{Dr}=0.0$ in addition to $\mathrm{Dr}=0.49$ are computed and compared with measured results (Fig.15). For a simple meandering channel $(\mathrm{Dr}=0.0)$, we can see large scours along the outer bank. However, the increment in the relative depth of a compound meandering channel causes inner bank scours instead of the outer bank scour. The reason of the above phenomena is that the helical flow structure in a compound meandering channel is different from that in a simple meandering channel due to $3 \mathrm{D}$ 


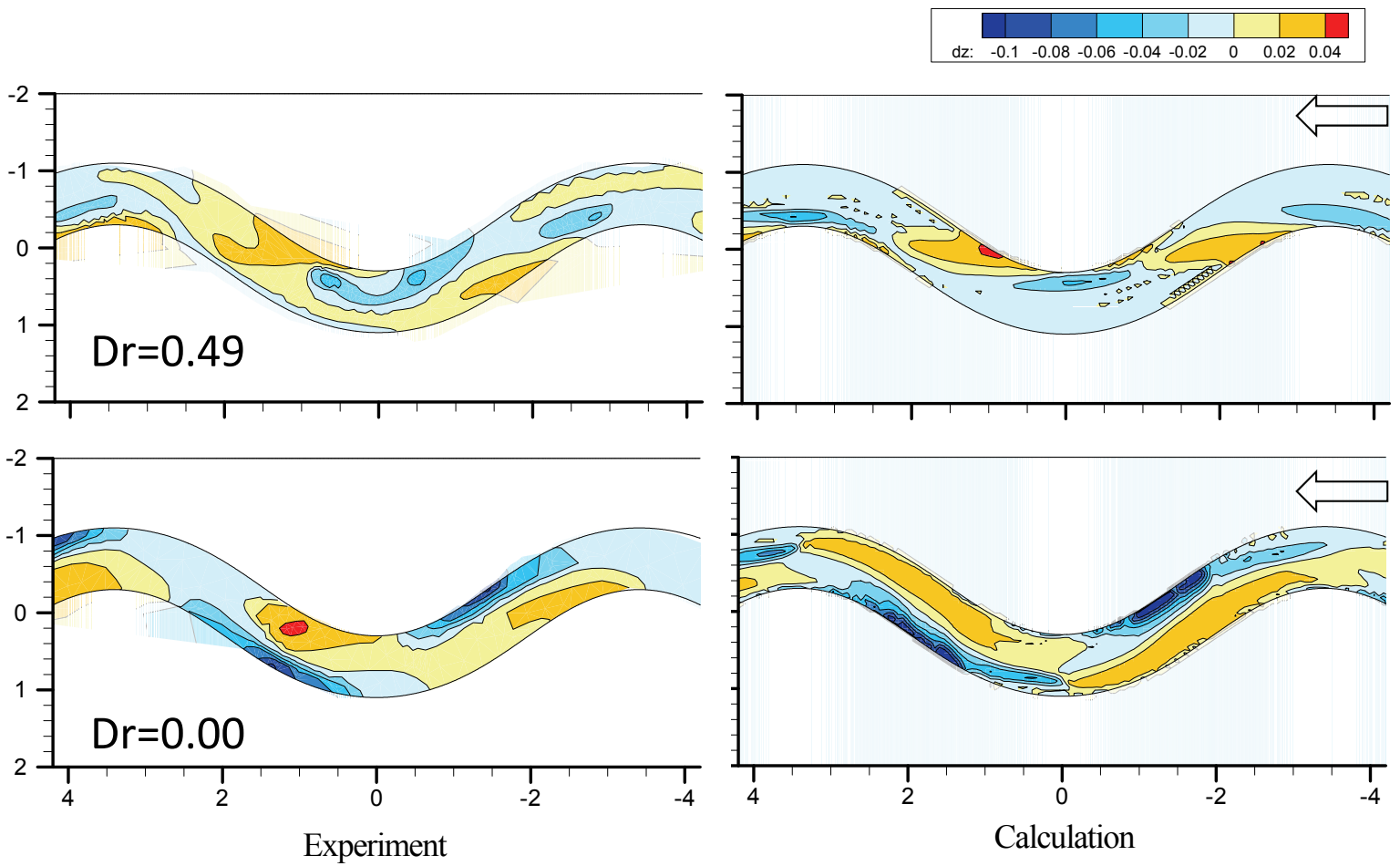

Fig.15 Bed variations in compound meandering channel for two different types of flow

flow interactions between a main-channel and flood plains. The present calculation results give a better agreement with experimental results rather than full-3D calculation results with the periodic boundary condition ${ }^{29)}$. This proves that the actual boundary conditions should be given for the bed variation analysis, because the periodic boundary condition would give an unrealistic assumption for the analysis. Therefore it would be safe to say that the general BVC method can reproduce bed variations in a compound meandering channel flow with increasing relative depth.

\section{BED VARIATION ANALYSIS USING OBSERVED TEMPORAL WATER LEVEL PROFILES IN RIVER DURING A LARGE FLOOD EVENT}

It is difficult to know temporal changes in bed elevations during the period of floods because of the difficulty of measurement. We have developed the calculation method with observed temporal changes in water surface profiles based on the idea that the influences of channel shape and resistance are reflected in temporal changes in water surface profiles ${ }^{5,30)}$. The influence of bed variation during flood events is also reflected in temporal changes of water surface profiles in the section where the large degree of bed variation occurs. The above indicates that the bed variation during a flood event would be

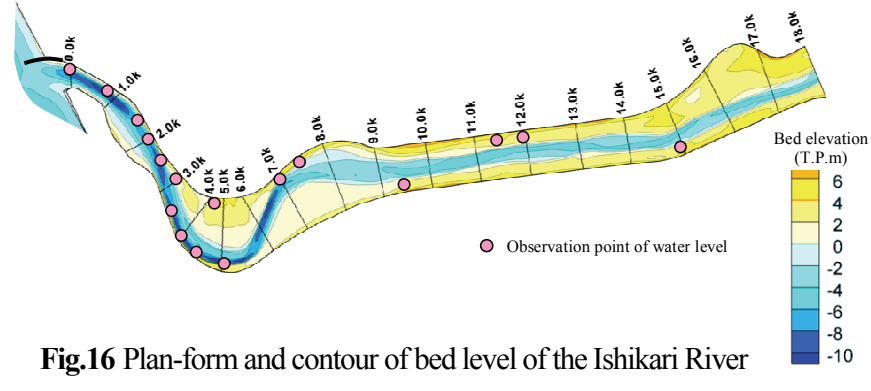
mouth and observation points of 1981 flood

clarified by using a suitable calculation method. This section demonstrates the bed variation analysis during the August 1981 large flood of the Ishikari River.

The peak discharge of the flood at the Ishikari River mouth exceeded the design discharge and a large bed scouring occurred in the river mouth. During the flood, temporal data of water levels were measured at many observation points as shown in Fig. 16. The cross-sectional bed forms were surveyed before and after the flood. A lot of researches on the bed variation of the flood have been done. One of the important researches was conducted by Shimizu et al. ${ }^{31)}$ and Inoue et $a l^{32}{ }^{32}$ We developed the BVC method for flood flow with 2D bed variation analysis using observed temporal changes in water surface profiles. In this analysis, the concentration of suspended sediment was calculated by 3D advection-diffusion equations in order to evaluate suspended load affected by the secondary flow in meandering channel ${ }^{33), 34)}$.

Fig. 17 shows temporal changes in observed and calculated 


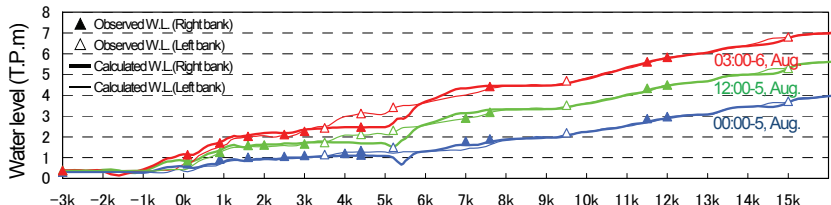

(a) Water level profiles during flood rising period

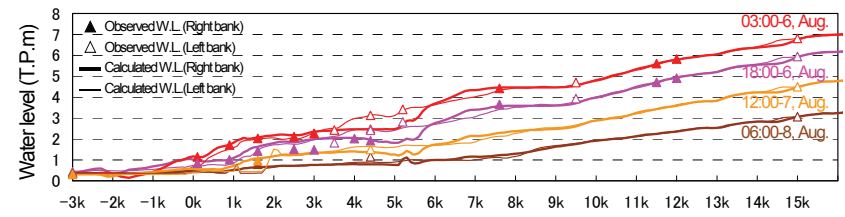

(b) Water level profiles during the flood falling period

Fig.17 Temporal changes in water surface profiles

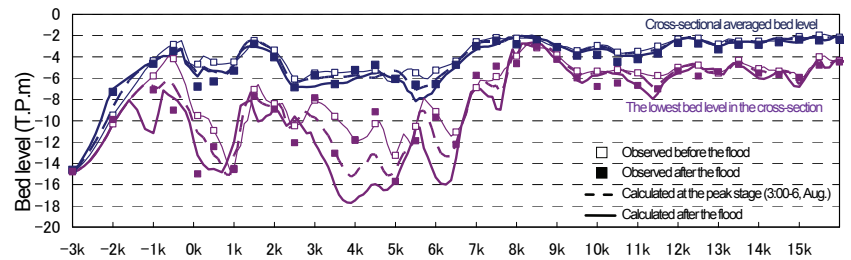

Fig.18 Longitudinal profiles of bed levels during the 1981 flood

water surface profiles in flood rising and falling periods. Calculated water surface profiles coincide with observed water levels affected by bed variations in each time and there are little differences between calculated and observed water levels.

Fig.18 shows the comparison between observed and calculated longitudinal bed forms before and after the flood. The crosssectional averaged bed elevations after the flood by the calculation are similar and nearly coincide with those of the observed result. Although the differences in the lowest bed levels at the cross-sections between measured and calculated results, large bed variations during the flood were explained by the calculation. Fig.19 shows the comparison of bed topographies in the Ishikari River mouth between calculated and observed results after the 1981 flood. It would be safe to say that calculated results capture the characteristics of observed bed topography in the meandering channel upstream from the Ishikari River mouth due to the large flood event.

\section{ISSUES AND FUTURE WORKS TOWARD INTEGRATED MULTI-SCALE SIMULATIONS}

As discussed in this paper, it is important for river channel designs and maintenance managements to elucidate flows and bed variations during floods by the reliable calculation method with use of observed data. The calculation method should be examined and developed so as to predict the actual phenomena of flows and sediment motions in rivers. The BVC method based on the depth integrated model can be applied to various flows as indicated in this paper, because the method is not

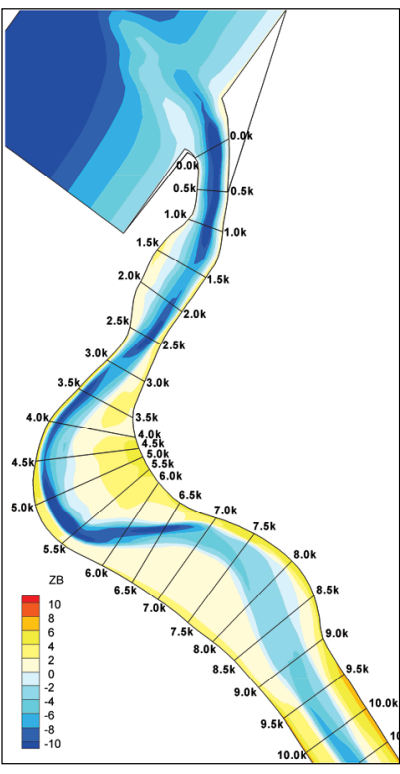

(a) Measured

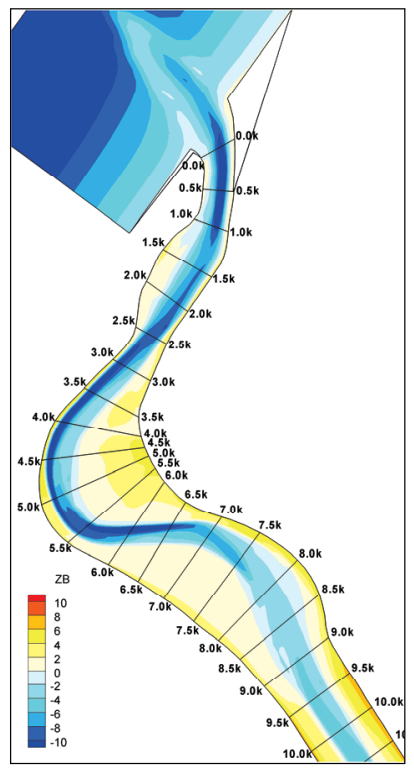

(b) Calculated
Fig.19 Comparison of bed topographies in the Ishikari River mouth between calculated and measured results after the 1981 flood

restricted by the shallow water assumption. However, the method is difficult to reproduce complex 3D flow dynamics such as separation zones behind structures. In relation to this issue, it is needless to say that researches on detail measurements in laboratory channels and developments of turbulence models would make an important contribution. On the other hand, the basic researches in laboratories are not always enough to clarify the large scale phenomena in rivers, because turbulence and vortex motions depend on the flow scale and vary with complex geometries. As discussed in the introduction, practical problems related to flows and sediment motions in rivers include various scale phenomena which affect each other. For example, Fig.20 and Fig.21 show comparisons of horizontal and helical flow structures along a vertical wall in the channel bend between the real scale field experiment and calculation by the general BVC method ${ }^{35)}$. For the section E, computed secondary flow intensity and streamwise velocity in the vicinity of the wall are relatively weak compared with those of the measurement. Main reasons are because the difference between the measurement and calculation in the approaching flow at the section $\mathrm{C}$ should be considered. The secondary flow in the channel bend is produced by the rotation and deformation of the lateral voriticity in the approaching flow. The approaching flow in the field experiment is affected by various scale factors such as resistance due to sediment particles with diverse sizes and shapes, complex bed topography, channel plan shape and hydraulic conditions at the upstream end. The above discussion indicates that a calculation method integrating these different effects is necessary for actual flood phenomena. Shimada et al. ${ }^{30,37)}$ have carried out full-scale experiments on 

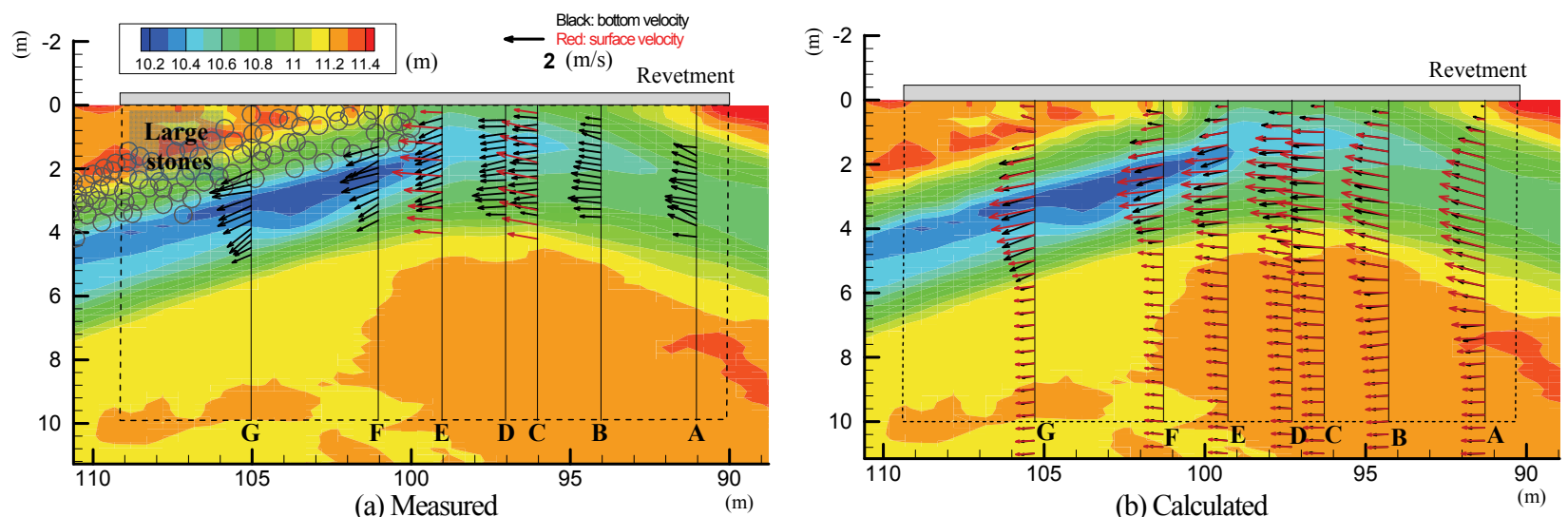

Fig.20 Comparisons of horizontal flow structures between the real scale experiment and the general BVC method ${ }^{35}$

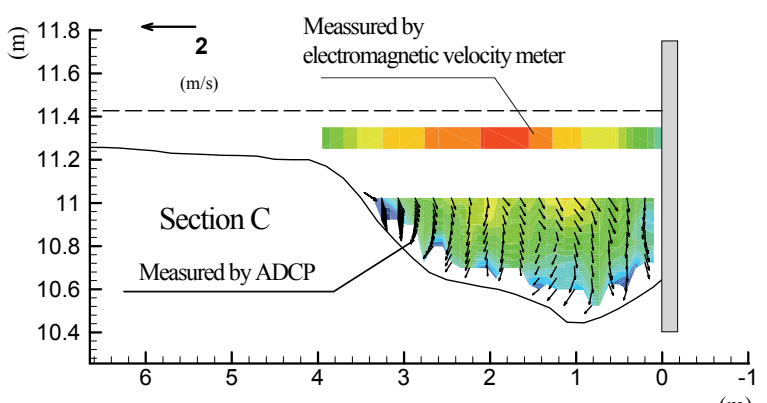

(m)

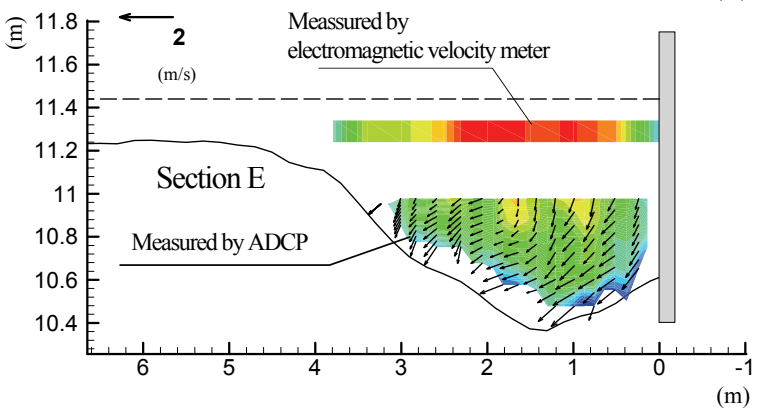

(a) Measured

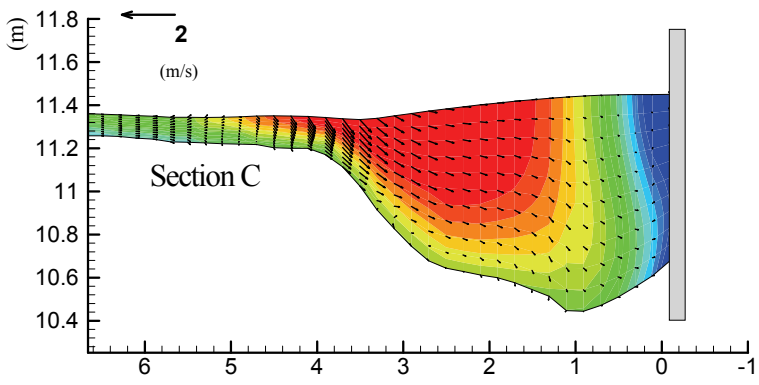

(m)
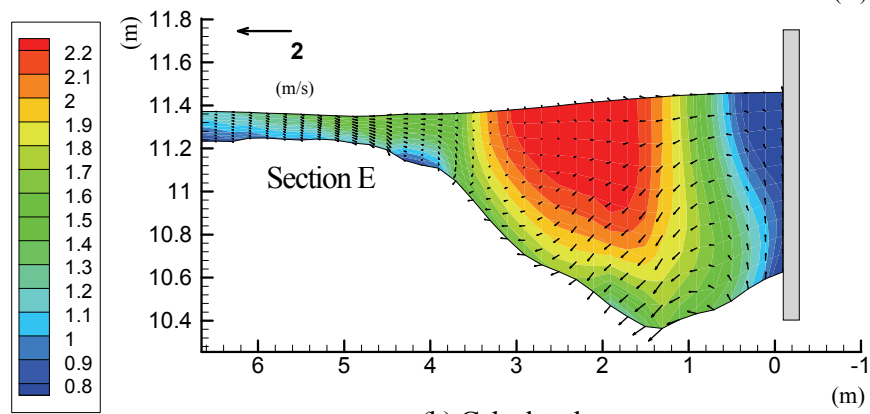

(b) Calculated

Fig.21 Comparisons of 3D flow structures between the real scale experiment and the general BVC method ${ }^{35)}$

levee breaches. Nihei and $\mathrm{Kimizu}^{38)}$ have developed a new monitoring system for the estimation of river discharge combining measured data and calculations. These researches are also expected for the clarification of actual flood phenomena. We should put forth a steady effort in measurements and analysis of the flood flows and bed variations in rivers together with basic hydraulic researches in the laboratory.

\section{REFERENCES}

1) Fukuoka, S.: Flood hydraulics and river channel design, Morikita Publishing Company, 2005.

2) Wu, W.: Computational river dynamics, Taylor \& Francis, London, 2008.

3) Shimizu, Y., Itakura, T. and Yamaguchi, H.: Numerical simulation of bed topography of river channels using two-dimensional model, Proceedings of the Japanese Conference on Hydraulics, Vol.31, pp.689-694, 1987.
4) Nagata, N., Hosoda, T. and Muramoto, Y.: Characteristics of river channel process with bank erosion and development of their numerical models, Journal of Hydraulic, Coastal and Environmental Engineering, JSCE, No.621/II-47, pp.11-22, 1999.

5) Fukuoka, S., Watanabe, A., Hara, T. and Akiyama, M.: Highly accurate estimation of hydrograph of flood discharge and water storage in rivers by using an unsteady twodimensional flow analysis based on temporal change in observed water surface profiles, Journal of Hydraulic, Coastal and Environmental Engineering, JSCE, 761/2-67, 45-56, 2004.

6) Engelund, F.: Flow and bed topography in channel bends, Journal of hydraulics division, Proc. of ASCE, Vol.100, HY11, pp.1631-1648, 1974.

7) Nishimoto, N., Shimizu, Y. and Aoki, K.: Numerical simulation of bed variation considering the curvature of stream line in a meandering channel. Proceedings of the Japan Society of Civil Engineers, Vol.456/II-21, pp.11-20, 1992.

8) Ikeda, S. and Nishimura, T.: Three-dimensional flow and bed topography in sand-silt meandering rivers, Proceedings of the 
Japan Society of Civil Engineers, Vol.369/II-5, pp.99-108, 1986.

9) Finnie, J., Donnell, B., Letter, J., and Bernard, R.S.: Secondary flow correction for depth-averaged flow calculations, Journal of Engineering Mechanics, ASCE, Vol.125, No.7, pp.848-863, 1999.

10)Blanckaert, K. and de Vriend, H. J.: Nonlinear modeling of mean flow redistribution in curved open channels, Water Resources Research, Vol.39, No.12, 1375, doi:10.1029/2003WR002068, 2003.

11)Onda, S., Hosoda, T. and Kimura, I.: Refinement of a depth averaged model in curved channel in generalized curvilinear coordinate system and its verification, Annual Journal of Hydraulic Engineering, JSCE, 50, pp.769-774, 2006.

12)Ishikawa, T., Suzuki, K. and Tanaka, M.: Efficient numerical analysis of an open channel flow with secondary circulations, Proc. of JSCE, Vol.375/II-6, pp.181-189, 1986.

13)Fukuoka, S., Watanabe, A. and Nishimura, T.: On the groin arrangement in meandering rivers, Journal of Hydraulic Engineering, JSCE, No.443/II-18, pp.27-36, 1992.

14)Jin Y.-C. and Steffler, P.M.: Predicting flow in curved open channels by depth-averaged method, J. Hydraul. Eng., ASCE, Vol.119, No.1, pp.109-124, 1993.

15)Yeh, K.-C. and Kennedy, J..F.: Moment model of nonuniform channel-bend flow. I: Fixed beds, J. Hydraul. Eng., ASCE, Vol.119, No.7, pp.776-795, 1993.

16)Ghamry, H. K. and Steffler, P. M.: Two dimensional vertically averaged and moment equations for rapidly varied flows, Journal of Hydraulic Research, Vol.40, No.5, pp.579587, 2002.

17)Ghamry, H. K. and Steffler, P. M.: Two-dimensional depthaveraged modeling of flow in curved open channels, Journal of Hydraulic Research, Vol.43, No.1, pp.44-55, 2005.

18)Uchida, T. and Fukuoka, S.: A bottom velocity computation method for estimating bed variation in a channel with submerged groins, Journal of JSCE, Ser. B1 (Hydraulic Engineering), Vol.67, No.1, pp16-29, 2011.

19)Uchida, T. and Fukuoka, S.: Bottom velocity computation method by depth integrated model without shallow water assumption, Journal of JSCE, Ser.B1 (Hydraulic Engineering), Vol. 68, No. 4, I_1225-I_1230, 2012.

20)Uchida, T. and Fukuoka, S.: A computation method for flow over structures, Advances in River Engineering, Vol.18, pp.351-pp.356, 2012.

21)Uchida, T. and Fukuoka, S.: Numerical simulation on sand waves using depth integrated model without the shallow water assumption, Journal of JSCE, Ser.B1 (Hydraulic Engineering), Vol. 69, No. 4, 2013, accepted.

22)Hashimoto, H. and Fujita, K. et al: Investigations on the overtopping levee, Technical Note of PWRI, No.2074, 1984.

23)Giri, S., Shimizu, Y.: Numerical computation of sand dune migration with free surface flow, Water Resources Researches, Vol.42, W10422, doi:10.1029/2005WR004588, 2006.

24)Niemann, S.L., Fredsøe, J. and Jacobsen, N.G.: Sand dunes in steady flow at low Froude numbers: dune height evaluation and flow resistance, J. Hydrauic. Eng., Vol.137, No.1, pp.5-14,2011.

25)Onda, S. and Hosoda, T.: Numerical simulation on development process of dunes and flow resistance, Annual Journal of Hydraulic Engineering, JSCE, 48, 973-978, 2004.
26)Fukuoka, S., Okutsu, K. and Yamasaka, M.: Dynamic and kinematic features of sand waves in upper regime, Proceedings of JSCE, No.323, 1982.

27)Roulund, A., Sumer, B. M., Fredsøe, J. and Michelsen, J.: Numerical and experimental investigation of flow and scour around a circular pile, Journal of Fluid Mechanics, Vol.534, pp.351-401, 2005.

28)Fukuoka, S., Omata, A., Kamura, D., Hirao, S. and Okada, S.: Hydraulic characteristics of the flow and bed topography in a compound meandering river, Journal of Hydraulic, Coastal and Environmental Engineering, JSCE, No.621/II47, pp.11-22, 1999.

29)Watanabe, A. and Fukuoka, S.: Three dimensional analysis on flows and bed topography in a compound meandering channel, Annual Journal of Hydraulic Engineering, JSCE, 43, 665-670, 1999.

30)Fukuoka, S.: What is the fundamentals of river design utilization of visible techniques of sediment laden-flood flows, Advances in River Engineering, JSCE, Vol.17, pp.8388, 2011.

31)Shimizu, Y., Itakura, T., Kishi, T. and Kuroki, M.: Bed variations during the 1981 August flood in the lower Ishikari River, Annual Journal of Hydraulic Engineering, JSCE, Vol.30, 487-492, 1986.

32)Inoue, T., Hamaki, M., Arai, N., Nakata, M., Takahashi, T., Hayashida, K., and Watanabe, Y.: Quasi-three-dimensional calculation of riverbed deformation during 1981-year flood in the Ishikari River mouth, Advances in River Engineering, Vol.10, 101-106, 2004.

33)Okamura, S., Okabe, K. and Fukuoka, S.: Numerical analysis of quasi-three-dimensional flow and bed variation based on temporal changes in water surface profile during 1981 flood of the Ishikari River mouth, Advances in River Engineering, Vol.16, pp.125-130, 2010.

34)Okamura, S., Okabe, K. and Fukuoka, S.: Bed variation analysis using the sediment transport formula considering the effect on river width and cross-sectional form -in the case of 1981 flood of the Ishikari River mouth-, Advances in River Engineering, Vol.17, pp.119-124, 2011.

35)Koshiishi, M., Uchida, T. and Fukuoka, S.: A new method for measuring and calculating three-dimensional flows and bed forms around river banks, Journal of JSCE, Ser.B1 (Hydraulic Engineering), Vol. 69, No. 4, 2013, accepted.

36)Shimada, T., Watanabe, Y., Yokoyama, H., and Tsuji, T.: Cross-levee breach experiment by overflow at the Chiyoda Experimental Channel, Annual Journal of Hydraulic Engineering, JSCE, Vol.53, pp.871-876, 2009.

37)Shimada, T., Hirai, Y. and Tsuji, T.: Levee breach experiment by overflow at the Chiyoda Experimental Channel, Annual Journal of Hydraulic Engineering, JSCE, Vol.54, pp.811-816, 2010.

38)Nihei, Y. and Kimizu, A.: A new monitoring system for river discharge with an H-ADCP measurement and riverflow simulation, Doboku Gakkai Ronbunshuu B, Vol.63, No.4, pp.295-310,2007.

(Received December 31, 2012) 